\title{
A Study of Variations in the Branching Pattern of Splenic Artery
}

\author{
Dr. Balachandra $\mathrm{N}^{1}$, Dr. B R Ramesh ${ }^{2}$ \\ ${ }^{I}$ Associate Professor, Department Of Anatomy, $\operatorname{Dr} B R$ Ambedkar Medical College, $K$ G Halli, Bengaluru \\ ${ }^{2}$ Professor And Head, Department Of Anatomy, Dr B R Ambedkar Medical College, K G Halli, Bengaluru
}

\begin{abstract}
Splenic artery is the largest branch from the celiac trunk supplying the spleen, pancreas and the stomach.. It divides into two or three main branches before entering the hilum of the spleen. 21 splenic arteries were studied by dissection method and 2 splenic arteries showed variation in their branching pattern described in the observations. In the cadavers studied, the splenic artery was arising from the celiac trunk. Of the 21 splenic arteries studied, 2 had variant branching pattern while the rest were showing normal distribution. One was in a male cadaver \& the other was in a female cadaver. Knowledge of variation in the branching pattern of the splenic artery is of importance during surgical and radiological procedures of the upper abdominal region to avoid any catastrophic complications.
\end{abstract}

Keywords: Splenic artery, variation in branching pattern, infarction

\section{I.Introduction}

Splenic artery is the largest branch from the celiac trunk supplying the spleen, pancreas and the stomach. It is tortuous from its origin and lies in multiple loops or even coils which appear above the superior border of the pancreas and descend to lie behind the gland. It divides into two or three main branches before entering the hilum of the spleen. As these branches enter the hilum they further divide into four or five segmental arteries that each supply a segment of the splenic tissue. There is relatively little collateral circulation between the segments implying infarction of that part of the spleen whose segmental artery becomes occluded.

\section{Materials and Methods}

Formalin embalmed cadavers given to IMBBS students for dissection. 21 splenic arteries were studied for the variation in their pattern, by dissection method.

\section{Results and Discussion}

In the cadavers studied, the splenic artery was arising from the celiac trunk. Of the 21 splenic arteries studied, 2 had variant branching pattern while the rest were showing normal distribution. One was in a male cadaver \& the other was in a female cadaver. The observations are as follows;

In the male cadaver aged about 60 yrs., given to undergraduate students for dissection, the splenic artery was seen to be branching early, about 2.5 inches from the hilum of the spleen. It was seen giving a superior branch and an inferior branch. These branches anastomosed about 1 inch in front of the hilum and subdivided into 5 branches before entering the spleen. The inferior polar branch was subdividing into 2 branches. In addition the left gastroepiploic artery was seen arising from the inferior branch.

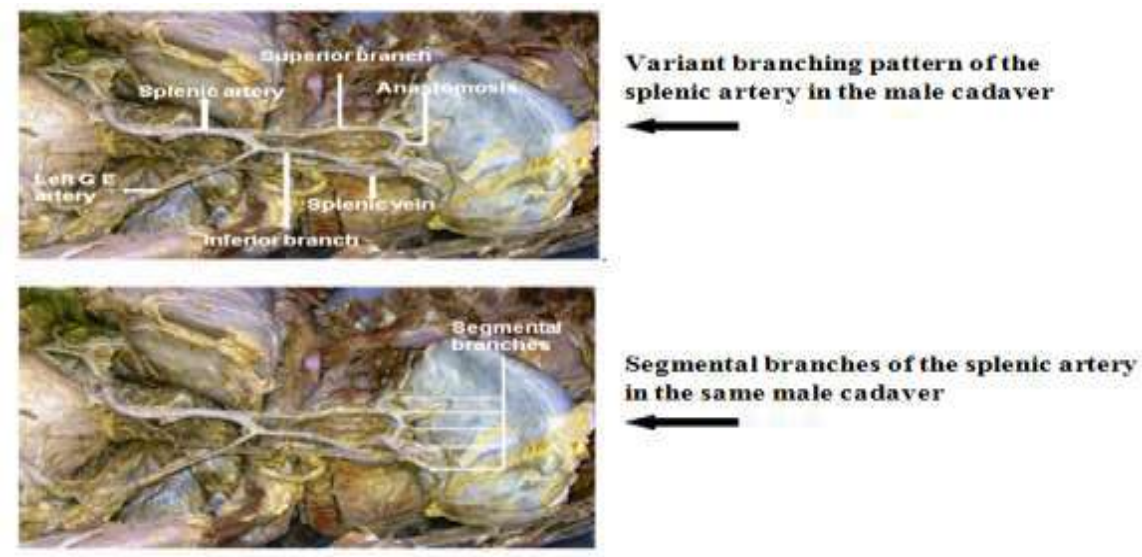




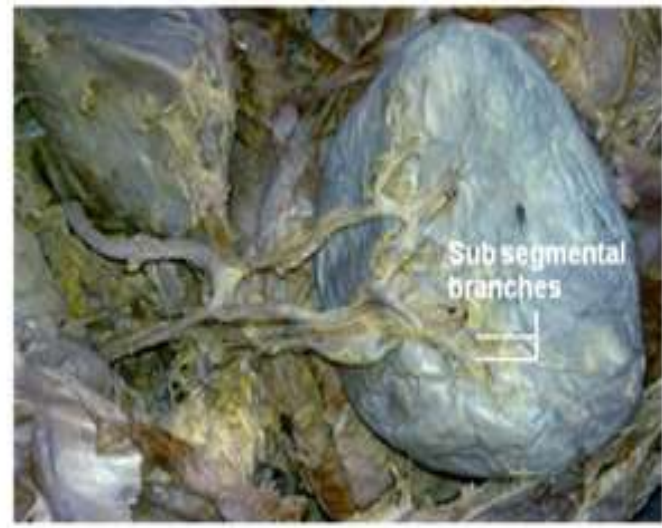

\section{Sub segmental branches of the splenic artery in the same male} cadaver

In a female cadaver aged about $80 \mathrm{yr}$, given to IMBBS students for discussion, we found the splenic artery giving off a superior polar branch. The main artery is giving off upper $\&$ lower terminal branches. These terminal branches are giving off segmental branches; three from upper \& two from lower, respectively, before entering the hilum. The main artery continues as the gastroepiploic artery \& from it, the lower polar artery is arising. The spleen also has lobulations \& is segmented

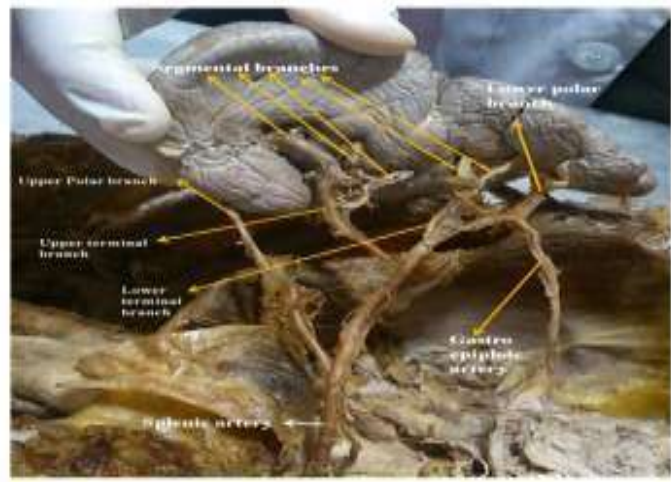

Nicholas A. Michels (1942) studied 100 splenic arteries. According to him no two splenic arteries were similar in pattern. He described 2 types of branches; Type 1- Prepancreatic (70\%) \& Type 2-Prehilar (30\%). Chen Weipei et al (1982-02) described 3 types of branching in dog spleens Type 1 - Biramification (89\%); Type 2 - Triramification (08\%); Type 3 - Polyramification (03\%). In type 1, they observed that most of the splenic arteries divide into superior and inferior splenic lobar arteries. Further, most of the superior splenic lobar arteries subdivided into the superior and mid-superior segmental arteries and the inferior splenic lobar artery subdivided into the mid-inferior and inferior segmental arteries. They were of the opinion that all of the splenic arteries of the dogs may divide into two splenic lobar arteries and each lobar artery further divides into two segmental arteries without exception. They observed poorly vascularized zones between the lobar or segmental arteries. Based on the anatomic observations they had performed experimental partial splenectomies on 15 dogs. All of the dogs survived the operation and their wound made on the spleen healed up very well.

Ronald A. Bergman et al (1981). Described 2 types of splenic arterial terminations: 1 . Magistral - 1-2cm from the hilus(30\%). 2. Distributing - Earlier branching (70\%)

Seok Kil Zeon et al (1998) studied 43 angiographic patterns of splenic artery and 7 celiac arteriograms of 36 males and 14 females aged between 23-67yrs. They observed the following branching pattern of splenic artery: $76 \%$ Superior polar; $24 \%$ Inferior polar; $100 \%$ terminal sup and inf branches; $28 \%$ terminal media arteries. A Daisy Sahni et al (2003) studied 200 adult autopsy specimens. They observed the Splenic artery dividing into 2-3 lobar arteries. Each lobar artery subdivided into 2-4 lobular arteries. 6-12 lobular arteries entered the spleen at its hilum. Polar arteries arose quite proximal to the hilum in $51 \%$.

S.K. Pandey et al (2004) observed the splenic artery dividing into terminal branches in 311 (97\%) cadavers. In nine $(2.8 \%)$ cadavers it passed through the hilum of spleen without dividing. Two terminal branches were the most common $(63.1 \%)$ followed by four $(18.8 \%)$, six $(9.7 \%)$, and more than six $(5.6 \%)$ branches. They concluded that there is variation in origin, course, and terminal distribution pattern of the splenic artery.

Present case (2011 - 2016) in the male cadaver, the superior and inferior splenic arteries arose 2.5in. 
from the hilus of the spleen. They anastomose about 1/2in. From the hilum and divide into 5 branches entering spleen at or near its hilum. In the female cadaver the main artery is giving off upper \& lower terminal branches. These terminal branches are giving off segmental branches; three from upper \& two from lower, respectively, before entering the hilum. The main artery continues as the gastroepiploic artery $\&$ from it, the lower polar artery is arising. The spleen also has lobulations \& is segmented. Embryological basis of vascular variations: The variations in the arterial patterns developed in the splenic artery could be due to; (1) Divergence in the mode of and proximo-distal level of branching; (2) Presence of unusual compound arterial segments; (3) Aberrant vessels that connect with principal vessels, arcades or plexuses

\section{Conclusion}

Variation in the course and termination of the splenic artery has been described frequently. Knowledge of these variations are of utmost importance as splenic artery interventions are increasingly performed to treat various clinical conditions, including abdominal trauma, hypersplenism, splenic artery aneurysm, portal artery hypertension, and splenic neoplasm. When clinically appropriate these procedures may provide an alternative to open surgery. They may help to salvage splenic function in patients with posttraumatic injuries or hypersplenism and to improve hematologic parameters in those who otherwise would be unable to undergo high - dose chemotherapy or immunosuppressive therapy. Also, the knowledge of these branching patterns indicate that in some spleens there will not be any infarction because of the multiple branching in the substance of the spleen as observed in some studies.

Conflict of interests: None

\section{Acknowledgements}

We are thankful to our colleagues in the department Drs Vasudha Kulkarni, Poonam D N and Shylaja D K, Assistant Professors and Mr Arul Sakthi, Mr Kiran Kumar, Tutors and the non teaching staff.

\section{List of References}

[1]. Nicholas A. Michels, The variational anatomy of the spleen \& the splenic artery, American Journal of Anatomy, vol 70, (1), p 2172, Jan 1942

[2]. Chen Weipei et al, Observations on the applied anatomy of the splenic artery, Acta Anatomica Sinica, $1982-02$.

[3]. Ronald Bergman et al, Compendium of Human Anatomic Variations: Catalog Atlas \& World literature.Urban \& Schwarzenberg, Baltimore \& Munich, 1988.

[4]. Seok Kil et al, Angiographic Branching Patterns of the splenic artery, International Journal of Angiology, 7: 575.

[5]. A Daisy Sahni et al, Branches of the Splenic artery and splenic arterial segments, Clinical Anatomy (N Y) 10/2003; 16(5): $371-7$.

[6]. Susan Standring, Gray's Anatomy; The Anatomical Basis of Clinical practice, 40th edition, Elsevier Churchill Livingstone; 2005, pp. 1480.

[7]. Pandey S, Bhattacharya S, Mishra R. and Shukla V. (2004), Anatomical variations of the splenic artery and its clinical implications. Clinical Anatomy, 17: 497-502.

[8]. David C Madoff et al, Splenic Arterial Interventions: Anatomy, Indications, Technical Considerations \& Potential Complications,

[9]. RadioGraphics, The journal of continuing medical education in radiology, April 25, 2005. 